\section{Archaeopteryx palaeontological myopia}

SIR - Howgate' criticizes our interpretations ${ }^{2,3}$ of the homologies and morphology of the Archaeopteryx manus by distorting and misrepresenting our viewpoints. Palaeontologists have identified the digits of theropods, Archaeopteryx and birds as digits 1-2-3 of the primitive reptilian hand whereas embryologists ${ }^{4-7}$ have identified the bird digits as 2-3-4. Do the similarities observed by palaeontologists have greater weight than the evidence derived from ontogeny by embryologists?

The correspondence of the number of phalangeal elements in the manus of $\boldsymbol{A r}$ chaeopteryx with the primitive reptilian formula has convinced palaeontologists that the digits of Archaeopteryx represent the digits 1-2-3. We stated that the digits of birds must be 2-3-4 based on embryological work ${ }^{4,5}$. If Archaeopteryx is a bird, it should have digits 2-3-4 and not 1-2-3 as suggested by Howgate ${ }^{1}$.

Howgate ${ }^{1}$ states that we $^{2,3}$ resurrected " a broken phalanx hypothesis" proposed by Heinroth ${ }^{8}$ which states that there was a break in the proximal phalanx of topographic digit 3 and that we "arrived at a non-theropod phalangeal formula (2-3-3)"'. We cited Heinroth ${ }^{8}$ because it was essential for a complete review. We stated that "The break or joint in the first phalanx of the fourth digit appears immediately distal to the immobilized area". We again stated ${ }^{3}$ (p.144) that "either a break occurred in the first phalanx (of the last digit) or if there are two phalanges a distortion of their orientation". We did not conclude that the phalangeal formula of Archaeopteryx was 2-3-3, but only stated the possibility of there being a break. Contrary to Howgate ${ }^{\prime}$, only the Berlin and Maxberg specimens have the essential areas of the manus preserved as bone. Their state of preparation precludes a definitive statement of the phalangeal formula of $A r$ chaeopteryx.

In the left manus of the Berlin specimen there is a laterally directed flange on the first phalan $x$ of the middle digit, directed to the junction of the supposed joint or break. Howgate implied that $w^{2}$ said that this flange "served to brace and strengthen the wing" ". Howgate' further states that "the 'flange' appears to be a secondary outgrowth due to injury or disease and not an original morphological structure". We did not state that this flange could serve to brace the wing since such a small structure cannot brace an entire wing. This functional misconception can only be attributed to Howgate. His illustration of the right manus demonstrates that this area is covered by matrix and a digit. Therefore how can he conclude the flange is an anomaly or a normal structure?

Attempting to make our explanation appear ludicrous, Howgate ${ }^{\prime}$ implies that our "scenario" of the preservation of the fourth digit (its crossing under the third digit) was the result of Archaeopteryx "crossing its fingers hopefully as it plunges at high speed in the sea wing-tips first". We stated (as a scenario) that the break or joint in topographic digit 3 was caused by forces during stalling 9,10 , impact upon the water, or as a result of preservation. Howgate ${ }^{1}$ also stated that "all the specimens of $A r$ chaeopteryx with a well preserved manus have the second and third digits crossed"' This is not the case since the Maxberg specimen ${ }^{11}$ has a manus without this condition.

The important aspect of the homologies of the avian manus is the debate between the 1-2-3 and 2-3-4 hypotheses and the implication that different developmental patterns in digit reduction can produce similar morphologies.

MAXK. HECHT SAMUALF. TARSITANO

Department of Biology,

Queens College of the City University of New York,

Flushing, New York 11367, USA

1. Howgate, M. Nature 306, 644 (1983).

Tarsitano, S. \& Hecht, M.K. J. Linn. Soc, Zoo. 69, 149 (1980).

3. Hecht, M.K. \& Tarsitano, S. Geobios mem. spec. 6, 141 (1982)

Montagna, W. J. Morph. 76, 87 (1945)

Hinchliffe, J.R. in Vertebrate Limb \& Somite Morphogenesis, 293 (1977).

6. Hinchliffe, J.R. \& Johnson, D.R. Dev. vert. limb. (Oxford, 1980)

Hinchliffe, J.R. \& Hecht, M.K. Evol. Biol, 16 (in the press). Heinroth, O. J. Om. 71, 277 (1923).

Nachtigall, W. Fortsch. Zool. 24, 13 (1977).

Pennycuick, C.J. Animal Flight (1972)

1. Heller, F. Erlanger Geol. Abhl. 31, 3 (1959)

\section{Evolutionary unease?}

SIR - Peter Fellgett raises an interesting suggestion' that the study of cybernetic feedback loops throws light on the redundant (multiple copy) nature of functional genes and hence places the evolution of this phenomenon within the rubric of Darwinian natural selection. His proposition is that selection has favoured organisms in which gene redundancy not only protects them from mutation but also leads to enhanced prospects for survival in that 'a new [biological] program would run rather than crash'. He considers this system of 'selection for selectability' as more acceptable than so-called alternative ad hoc suggestions such as molecular drive, which he thinks should only be invoked as additional evolutionary mechanisms if the necessities were demonstrated; the impliction being that selection is our basic null hypothesis.

I have no objection to Fellgett's basic proposition, although I cannot understand his premise that the scientific necessity for alternative mechanisms depends on which side of the Darwinian fence they fall. Molecular drive ${ }^{2}$ is based on several well-known processes of non-reciprocal exchange between redundant genes, all of which have the interesting and biologically important effect of continually replacing a family of genes with newly-arising mutant genes, throughout a sexual population. This mechanism of spreading a mutant gene, or parts of genes, can explain the widespread observation of clear differences between species in the way in which families have become replaced (homogenized). The phenomenon has been observed in families of both protein-coding and RNA producing genes and in non-functional DNA families, generally irrespective of the degree of redundancy or chromosomal dispersion of the families. Hence it is extraordinarily difficult to explain by selection alone the parallel homogenization of hundreds of families and the accidental gain and loss of complete subfamilies and families, across any one species, without invoking generally implausible ad hoc scenarios. Genetic turnover potentially constitutes an independent mechanism of population change with respect to both the de novo origin and the subsequent behaviour of redundant genes. Selection and drift can be expected to interact with this mechanism, depending on the gene family and population structure in question (see refs 2-9).

I am quite comfortable with Fellgett's idea that the origin of redundancy (and I would add the existence of nonreciprocal exchange processes within the redundant families) might be due to selection via cybernetic multiple feedback loops, that is, the processes underlying molecular drive have arisen via natural selection. There is nothing in evolution that cannot be explained by natural selection, if that is our starting point. However, this possibility would need to take into account why so many hundreds of qualitatively different genic and non-genic DNA families exist, why the degree of redundancy varies over several orders of magnitude both for the same family between species and for different families in the same species, why many families differentiate into multiple subfamilies, and why there are continual rounds of family homogenization by variant genes or parts of genes across a species. An unsubstantiated appeal to a seeming Darwinian "selection for selectability" based on cybernetic theory is not a sufficient justification for discounting the necessity for an additional evolutionary mechanism which is based on known DNA turnover mechanisms within the genome. That molecular drive is non-Darwinian is surely irrelevant.

GABRIEL.A. DOVER

Department of Genetics, University of Cambridge, Cambridge CB2 3EH, UK

Fellgett, P. Nature 308, 230 (1984)

Dover, G.A. Nature 299, 111 (!982)

Ohta, T. Lecture Not. Biomalh. 37. (1980).

Arnhcim, N. in Evolution of Genes \& Proteins (cds Nci, M. \& Koehn, R ) 38 (Sinauer, Boston, 1983).

Hood, 1. et al. A. Rev. Genet. 9, 305 (1975).

Fcdoroff, N. Cell 16, 697 (1979).

Kedes, L..H. A. Rev. Biochem. 48, 837 (1979).

Kedes, I..H. A. Rev, Biochem. 48,837 (1979).
Flavell, R.B. in Genome Evolution (eds Dover, G.A. \& Flavell, R.B. in Genome Evolution (eds Do
Flavell, R.B.) 301 (Academic, London, 1982).

Jeffreys, A. in Genome Evolution (eds Dover, (i,A. \& Flavell, R.B.) 15? (Academic, London, 1982). 